patient; messages may not have been received. As the initial response to more regular follow up was positive, consideration should be made to whether a pre-arranged phone clinic would be more suitable. Future research could focus on reasons why $\mathrm{CYP} /$ their families are reluctant to engage, and ways to empower CYP to change.

To pave the way forward a patient questionnaire evaluating the current service and seeking opinions regarding regular, remote follow up would be valuable.

\section{P18 EFFECTIVENESS OF HOME BOWEL PREPARATION FOR CHILDREN LIVING LONG DISTANCES FROM ENDOSCOPY CENTRE}

Jamie Motion, Malcolm Sutherland, Brenda Smart, Carol Cameron, Shyla Kishore, lain Chalmers, Su Bunn. NHS Grampian

\subsection{6/flgastro-2021-bspghan.28}

Introduction The North of Scotland Paediatric Gastroenterology, Hepatology and Nutrition Network (NoSPGHANN) manages children over an area of $53,000 \mathrm{~km}^{2}$. Travel distances to Royal Aberdeen Children's Hospital (RACH) were previously felt to preclude the adoption of home bowel preparation (HBP) for elective colonoscopies but a trial period of HBP commenced in March 2020. The same drugs (senna and Picolax) were used for inpatient bowel preparation (IPBP) or HBP but the timings were changed for HBP to complete all doses on the day prior to procedure to allow travel to RACH. This audit evaluates the impact of this change of practice.

Methods All children undergoing elective colonoscopy at RACH between December 2019 and November 2020 were identified. Electronic were records reviewed to determine IPBP vs HBP, distance to RACH from patient's home, bowel preparation score, morning or afternoon list, requirement for intravenous (IV) fluids during the procedure, day case procedure and length of stay. Bowel preparation score was derived from the Aronchick Scale and converted as follows: 0 (unacceptable), 1 (poor), 2 (fair), 3 (good) and 4 (excellent).

Results

Summary The high standard of bowel preparation achieved with IPBP was maintained when delivered at home, despite some children travelling $>100$ miles and having travelling times of $>3$ hours. Delivering all doses of drugs on the day before procedure did not affect the quality of bowel preparation for afternoon lists. There is a trend to a higher proportion of children with HBP receiving IV fluids during anaesthetic which may suggest that some are dehydrated. The proportion of day case procedures has increased from $0 \%$ to 72\%, which since March 2020, has saved NHS Grampian $£ 18,000$.

Conclusion Home bowel preparation delivered on day prior to procedure is well tolerated and as effective as inpatient delivered, even for children with long travelling times to hospital. Covid-19 distancing measures have reduced the number of available inpatient beds so HBP has aided bed management in addition to providing a cost saving. The risk of dehydration may be higher for HBP and guidance will be changed to increase the emphasis on oral fluid intake, including during travelling time, on day of procedure.

\section{P19 EFFICACY OF THIOPURINES IN PREVENTING INFLIXIMAB ANTIBODY FORMATION WHEN USED IN DUAL THERAPY: EXPERIENCE FROM A SINGLE TERTIARY PAEDIATRIC GASTROENTEROLOGY DEPARTMENT}

Muhamad Azim Muhamad Amin, Varsha Datta, Danica Hapuarachchi, Loveday Jago, Andrew Fagbemi, Ahmed Kadir. Royal Manchester Children Hospital, Manchester

\subsection{6/flgastro-2021-bspghan.29}

Background and Aim There is evidence that shows addition of an immunomodulator (azathioprine or mercaptopurine) to Infliximab (IFX) therapy reduces antidrug antibodies, however, published evidence remains quite limited in paediatric population. We conducted a review to observe whether there is any correlation between the drug level of azathioprine metabolites, that is 6-thioguanine nucleotides (6-TGN) and development of anti-IFX antibodies (Abs) in inflammatory bowel disease (IBD) patients.

Method This is a retrospective study of patients with IBD based on a single tertiary paediatric gastroenterology department that had their levels monitored from March 2016 until March 2020. We defined maximum drug efficacy based on consensus on ESPGHAN management of IBD in paediatric and our lab references $(235-450 \mathrm{pmol} / 8 \times 108)$. In order to maintain consistencies, we included patients on 8 weekly 5 $\mathrm{mg} / \mathrm{kg}$ of Infliximab infusion regimen who had their azathioprine metabolites measured within 3 months from starting. Fishers test and Pearson correlation were used to test the correlation between the drug level of azathioprine metabolites and development of IFX Abs.

Results $36(58 \%)$ out of 62 patients were included in this study based on the above criteria (median age 14.25). Mean level of 6-TGN was lower in anti-IFX Abs-positive patients compared to anti-IFX Abs-negative patients (316.2 vs 322.8) with $6.607 \pm 57.51$ (CI $-123.5-110.3, \mathrm{p}=0.91)$. There is a positive correlation between positive Anti-IFX abs with lower level of Azathioprine metabolites with coefficient at 0.47 $(\mathrm{p}=0.05)$.

Conclusion Our data demonstrates there is positive correlation between lower levels of azathioprine metabolites and positive anti-IFX abs level, hence suggestive of the importance of adherence to treatment to ensure longevity usage infliximab in IBD patients.

\section{P20 EMERGENCI: A UK PROSPECTIVE SURVEY OF SEVERE GI BLEEDING (REQUIRING UPPER GI ENDOSCOPY) AND EMERGENCY ENDOSCOPY IN UNDER 16S}

${ }^{1}$ Natasha Thorn, ${ }^{1}$ Martina Vallorani, ${ }^{1}$ Ramiya Kirupananthan, ${ }^{2}$ Polychronis Kemos, ${ }^{2}$ Nicholas Croft. 'Paediatric Gastroenterology, Royal London Children's Hospital, Barts Health NHS Trust, London; ${ }^{2}$ Blizard Institute, Barts and the London School of Medicine, Queen Mary University of London

\subsection{6/flgastro-2021-bspghan.30}

Objectives EMERGENCi is a prospective, national, cohort study of emergency endoscopy and severe upper GI bleeds in children. Objectives were to produce national data of the clinical presentations, patient co-morbidities, indications, waiting times and endoscopic treatments for emergency endoscopy. Methods Units were identified through the UK Paediatric Gastroenterology and Paediatric Surgical Societies (BSPGHAN \& BAPS). Once registered, fortnightly emails were sent over a 6 month period asking for reports in $<16$ years of severe upper 